\title{
Almacenamiento de gas natural ${ }^{1}$
}

\author{
TOMÁs CoRREA ${ }^{2}$ \\ ElKin CASTRILlón ${ }^{3}$
}

\section{Resumen}

Las mayores reservas de gas natural en el mundo se encuentran en regiones remotas, alejadas de las grandes ciudades, siendo necesario diferentes alternativas para el transporte y almacenamiento del fluido, a través de gasoductos y barcos metaneros (CNG, LNG). Dependiendo de las distancias y los volúmenes se define cuál es el medio de transporte más viable.

En las regiones consumidoras de gas natural existen básicamente tres diferentes mercados; sector residencial y comercial, industrial y generación de electricidad. El sector residencial y comercial es altamente estacional con fuertes picos en el invierno, el de generación de electricidad varía, alcanzando picos en el verano cuando las temperaturas son muy altas; también existen factores externos diferentes al clima, como eventuales fallas o in-

1 Este es un estudio exploratorio al almacenamiento del gas natural en el mundo y hace parte del proyecto de Tesis de Maestría sobre Almacenamiento de Gas Natural en Colombia.

2 Ingeniero Mecánico de la Universidad Nacional de Colombia con maestría en ingeniería del gas natural de la Universidad de Oklahoma, USA. Actualmente es docente de tiempo completo del Instituto Tecnológico Metropolitano (ITM). Investigador del GITER (Grupo de Investigaciones en Tecnologías Energéticas). tomascorrea@itm.edu.co

3 Ingeniero en Instrumentación y Control del Politécnico Colombiano Jaime Isaza Cadavid con especialización en Gestión Energética Industrial del ITM. Actualmente es docente de tiempo completo del Instituto Tecnológico Metropolitano (ITM). Investigador del GITER (Grupo de Investigaciones en Tecnologías Energéticas). elkincastrillon@itm.edu.co 
terrupciones inesperadas en los sistemas de gasoductos (Europa principalmente). Esto implica para las compañías distribuidoras de gas natural, generar planes alternos que permitan disponer de gas natural para suplir los máximos consumos, picos de demanda y eventuales interrupciones durante el año. Uno de estos planes consiste en el uso de unidades de almacenamiento de gas en superficie o subterráneos de gran capacidad y de tasas de inyección y producción lo suficientemente altas para satisfacer las demandas del mercado. Las unidades de almacenamiento de gas subterráneo o en superficie son una opción como respaldo para garantizar el flujo continuo en situaciones de fallas o interrupciones inesperadas en el sistema de gasoductos nacionales.

Entre los diferentes tipos de unidades de almacenamiento de gas subterráneo existen tres tipos principales: yacimientos de petróleo o gas donde la producción no es económicamente viable (yacimientos agotados), cavernas de sal y yacimientos de agua o acuíferos. Ellos son adecuados de acuerdo con sus características geológicas y las necesidades de las compañías distribuidoras de gas natural. Este trabajo es una exploración a las características técnicas y económicas de los diferentes tipos de unidades de almacenamiento usados alrededor del mundo con la idea de abrir la discusión respecto a la pertinencia de sistemas de almacenamiento de gas natural en Colombia.

\section{Palabras clave}

Almacenamiento de gas natural, facilidades para hidrocarburos.

\section{Alostract}

The largest reserves of natural gas worldwide are found in regions far of main cities, being necessary different alternatives to transport the fluid to the consumption cities, such as pipelines, CNG or ships, LNG, depending on distances between producing regions and demanding regions and the producing volumes.

Consumption regions have three different markets to natural gas; residential and commercial, industrial and power generation 
sector. The residential and commercial is highly seasonal and power generation sector is quite variable depending on increases of temperature during summer time. There are also external issues that affect the normal gas flow such as fails on the national system or unexpected interruptions on it, what imply that companies which distribute natural gas should design plans that allow supplying the requirements above mentioned. One plan is using underground natural gas storage with capacities and deliverability rates enough to supply demands. In Colombia there are no laws in this sense but it could be an exploration to discuss different ways to store gas either way as underground natural gas storage or above superficies.

Existing basically three different types of underground natural gas storage; depleted reservoirs, salt caverns and aquifers. All of them are adequate according to geological characteristics and the needs of the distributors companies of natural gas. This paper is an exploration of technical and economical characteristics of different kind of storages used to store natural gas worldwide.

\section{Key words}

Underground natural gas storage, gas facilities. 


\section{INTRODUCCIÓN}

Las reservas actuales probadas de gas natural en el mundo son 6263 TCF [1] siendo suficientes para abastecer la demanda mundial en el mediano futuro. Para el año 2020 la producción de gas sobrepasaría la producción de crudo, en barriles equivalentes por año. Para esos tiempos, algunos países o regiones que actualmente poseen grandes reservas, muy probablemente pueden llegar a ser importadores o que de hecho ya son importadores, incluyendo Estados Unidos, Asia del Pacífico y la gran mayoría de países europeos. Esas reservas están en su mayoría en Rusia y el Medio Oriente lejos de las áreas de demanda lo que implica la necesidad de medios de transporte y/o almacenamiento de gas para garantizar el suministro continuo de las potenciales demandas en las regiones consumidoras en el corto y mediano futuro dadas las estadísticas arriba mencionadas.

La demanda por gas natural viene de tres principales mercados en el mundo; el sector domiciliario y comercial, el sector industrial y el sector de generación de electricidad. En los mayores centros de consumo la mayor demanda por gas natural es estacional; típicamente más gas es usado en meses de invierno para calentar los hogares en países con estaciones; de otro lado, en días de verano, cuando las temperaturas están por encima de los promedios las turbinas a gas (combine cycle) entran en operación como apoyo a los sistemas de generación de electricidad tradicionales para suplir el pico de demanda dado por un mayor número de unidades de aire acondicionado trabajando. El uso de turbinas a gas (combine cycle) es debido a que son flexibles en tiempos de apagado y de encendido (start up, start off). También existen demandas adicionales de gas natural durante el año diferentes al factor clima; la falta de soporte ante eventuales fallas o interrupciones inesperadas en los sistemas de distribución nacionales; estas interrupciones generalmente se presentan cuando un mismo gasoducto atraviesa más de un país. Este es el caso de los países europeos que dependen en gran parte del gas de Rusia, el cual atraviesa varios países (políticamente 
inestables o problemas fronterizos con Rusia). En el caso de Colombia, en el año de 1992, cuando se presentó el "apagón" no se contaba con un sistema de respaldo al sistema interconectado nacional basado en energía hidráulica, como consecuencia se presento un racionamiento de energía en todo el país, o recientemente ante el mantenimiento de los dos grandes centros productores (Cusiana-cupiagua y Chuchupa-ballenas) de gas natural del país, se presentan racionamientos para el sector industrial y de automóviles durante 3 y 4 días. El Estado colombiano menciona en dos leyes (artículo 90.2 de la ley 142 de 1994, decreto 1484 de 2005) algunas consideraciones respecto a garantizar la disponibilidad permanente y el orden de prioridad en emergencias como la mencionada anteriormente, pero no hay ninguna ley referente a sistemas de respaldo (unidades de almacenamiento de gas natural) en este sentido. Las compañías deben, por lo tanto, garantizar flujos continuos y suministros adicionales de gas por efectos climáticos o de respaldo ante eventuales fallas o interrupciones. Una alternativa es el uso de almacenes de gas subterráneos cerca de los grandes centros de consumo del combustible.

Históricamente las compañías distribuidoras y/o productoras principalmente en Estados Unidos y Europa han utilizado tres tipos de unidades de almacenamiento de gas natural subterráneos: Yacimientos agotados de hidrocarburos los cuales son adecuados aprovechando que ya son conocidas sus características geológicas y las propiedades de los fluidos, en un segundo nivel están las adecuaciones de formaciones subterráneas de sal que por su estructura permiten confinar volúmenes de gas fácilmente sin perdidas apreciables, además permiten altas tasas de inyección y de producción para ser usadas en tiempos cortos y en cualquier momento; por último, algunos yacimientos de agua (acuíferos) también son utilizados como unidades de almacenamiento de gas natural por tener geologías similares a los yacimientos agotados de hidrocarburos.

Este artículo es una exploración inicial al almacenamiento de gas natural en el mundo, describe los diferentes tipos de unidades 
de almacenamiento; yacimientos agotados [2], cavernas de sal [3] y acuíferos [2], sus características técnicas y económicas asociadas a ellos con la idea de que en Colombia se discuta la pertinencia o no del uso de unidades de almacenamiento de gas natural subterráneos o en superficie entre los diferentes actores de la cadena del gas natural en Colombia.

\section{Definiciones}

Capacidad: es el máximo volumen de gas que puede ser almacenado en una facilidad de acuerdo con su diseño. La capacidad de almacenamiento de gas es la suma del gas de trabajo más el gas base [4].

Gas base (cushion gas): La cantidad mínima de gas que la formación necesita para operar [5]; en otras palabras, es la cantidad de gas que permite la presión necesaria para producir el total de gas de trabajo.

Gas de trabajo (working gas): Se refiere a la capacidad total menos el gas base.

Tasa de inyección: Tasa a la cual el gas debe ser inyectado en la formación de acuerdo con consideraciones de diseño y operación.

Tasas de producción: Tasas a las cuales el gas será producido de acuerdo con los volúmenes y tiempos de demanda requeridos.

Ciclos: El número de veces que el volumen de gas de trabajo puede ser inyectado y producido en un año [6].

Porosidad: es el volumen de poros (espacio vacío) de una roca dividida por el volumen total de la roca [7]. Este es expresado en porcentaje y se denota por $\Phi$.

Permeabilidad: la permeabilidad absoluta es una propiedad intrínseca del medio poroso que expresa la capacidad que tiene el medio de permitir flujo a su través cuando está saturado $100 \%$ de una sola fase [8], expresado comúnmente en unidades Darcy. 
Factor de compresibilidad Z: Factor de desviación del gas, es la relación entre el volumen realmente ocupado por un gas a una presión y temperatura dada sobre el volumen que ocuparía ese mismo gas a la misma presión y temperatura si éste se comportara como un gas ideal.

\section{Almacenamiento del gaS}

Las diferentes formaciones geológicas adecuadas para almacenar gas han sido yacimientos agotados, acuíferos y cavernas de sal cercanos a las grandes regiones consumidoras de gas natural básicamente para reducir la necesidad de mayores infraestructuras en gasoductos (diámetros mayores), suplir los picos de demanda, garantizar consumos adicionales de los equipos de aire acondicionado en verano usando turbinas de gas para generar electricidad, suplir eventuales fallas o daños en los gasoductos; además de que actualmente cerca del $40 \%$ de los yacimientos de gas natural (stranded gas) están en regiones remotas, alejadas de las grandes ciudades, lo cual exige cubrir grandes distancias en transporte y disponerlo en sistemas de almacenamiento para garantizar el flujo continuo durante todo el año.

El uso de unidades de almacenamiento subterráneo de gas natural se originó a partir de 1915 cuando los yacimientos agotados estuvieron disponibles principalmente en los Estados Unidos [9] (Ohio, New York, California,) y Canadá [10], ellos fueron adecuados; taladrando más pozos (inyección, producción y observadores), instalando un sistema de distribución de gas superficial (gathering system), estaciones compresoras, medidores de gas, facilidades para la deshidratación de gas, así que grandes volúmenes de gas fueron producidos cada estación de invierno y nuevo gas era inyectado en el siguiente verano. Posteriormente, en 1946 fue adecuado el primer acuífero en Kentucky (EE.UU.), y para el año de 1961 estaría entrando en funcionamiento el primer domo de sal en Michigan (EE.UU.). 
En general cada tipo de unidad de almacenamiento de gas natural tiene sus propias características físicas, tales como porosidad y permeabilidad, y económicas como el sitio, costos de adecuación de la facilidad, mantenimiento, tasas de inyección y de producción, número de ciclos por año. Según las características geológicas se selecciona la estructura geológica más adecuada. Normalmente los yacimientos agotados son los mejores candidatos, debido al conocimiento que se tiene de la estructura geológica y fluidos almacenados en la misma, así como por las facilidades disponibles en superficie para el transporte y manejo de fluidos. En segunda instancia están los acuíferos, seguidamente las cavernas de sal y por último algunas minas abandonadas específicamente en EE.UU. (1), Bélgica (1), Alemania (1) y Francia (1) han sido usadas como unidades de almacenamiento subterráneas de gas natural. La figura 1 muestra la distribución de gas de trabajo por tipo de unidad de almacenamiento usados en el mundo. Allí se puede observar que alrededor del $81 \%$ del gas de trabajo usado como soporte de los sistemas de gasoductos en el mundo corresponden a yacimientos agotados. Un 14,5 \% corresponde a acuíferos debido a su cercanía en términos geológicos a los yacimientos agotados. El gas de trabajo proveniente de cavernas de sal corresponde a un 3,9\% del total caracterizadas por altas tasas de producción y al mayor número de

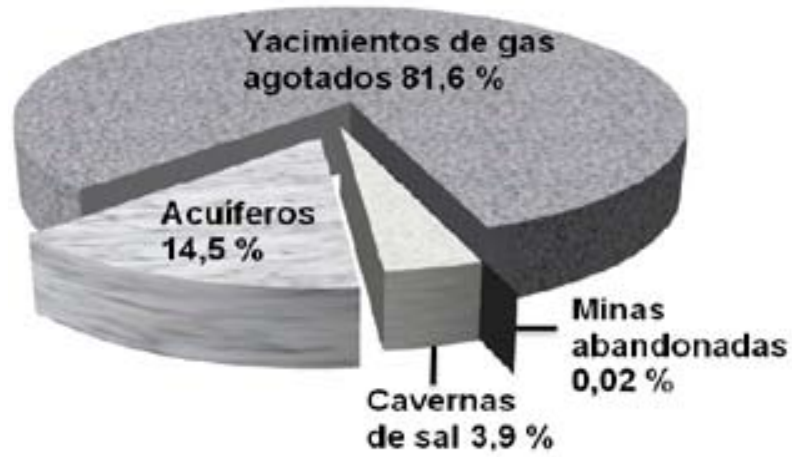

Figura 1. Distribución de VolÚMENES DE GAS DE TRABAJO POR TIPO DE UNIDAD DE ALMACENAMIENTO EN EL MUNDO [11] 
ciclos por año cuando es comparada con las otras dos alternativas, finalmente un $0,02 \%$ corresponde a minas abandonadas.

La tabla 1 muestra los volúmenes totales de gas base y de trabajo para las regiones de Norteamérica y Europa, así como el total de yacimientos adecuados como unidades de almacenamiento de gas y la capacidad de producción en billones de pies cúbicos por día [BCF/D] de acuerdo con sus existencias; sin embargo algunos países separadamente como Australia, posee 4 unidades de almacenes de gas subterráneo, Argentina 1 y China 1. Cabe resaltar que alrededor de un $50 \%$ del gas almacenado corresponde a gas base, el cual no será producido durante la vida del proyecto, por no ser rentable.

Tabla 1. Capacidades de almacenamiento [12]

\begin{tabular}{|c|c|c|c|c|}
\hline REGIÓN & $\begin{array}{c}\text { GAS DE } \\
\text { TRABAJO } \\
{[\mathrm{BCF}]}\end{array}$ & $\begin{array}{c}\text { GAS BASE } \\
{[\mathrm{BCF}]}\end{array}$ & $\begin{array}{c}\text { \# UNIDADES } \\
\text { DE ALMACENA- } \\
\text { MIENTO DE GAS } \\
\text { SUBTERRÁNEOS }\end{array}$ & $\begin{array}{c}\text { PRODUCCIÓN } \\
\text { BCF/D }\end{array}$ \\
\hline $\begin{array}{c}\text { Norte- } \\
\text { américa }\end{array}$ & 4506 & 4675 & 440 & 93 \\
\hline Europa & 6653 & 6590 & 168 & 83,4 \\
\hline
\end{tabular}

\subsection{Yacimientos agotados}

Este tipo de yacimientos son los primeros candidatos para ser adecuados como unidades de almacenamiento subterráneo de gas natural. El almacenamiento de este tipo viene determinado por el número de pozos de inyección/producción, por la capacidad de producción de los mismos y por el volumen de almacenamiento (porosidad). Los factores que determinan si un yacimiento agotado puede ser usado como unidad de almacén de gas dependen de la geología y la geografía. Geológicamente la roca debe tener una porosidad y una permeabilidad que garanticen una capacidad de almacenamiento y de producción acorde con los requerimientos de diseño y operación. Geográficamente, éste debe estar situado 
cerca de las grandes regiones consumidoras de gas natural para facilitar el suministro rápido, una vez éste sea requerido. En general, para este tipo de yacimiento, el gas base es alrededor de un $50 \%$, el periodo de inyección está entre 200 a 250 días y el periodo de producción fluctúa entre 100 y 150 días. En países de estaciones, generalmente es usado para suplir la sobre-demanda en las estaciones de invierno. La figura 2 muestra el número de yacimientos agotados adecuados como unidades de almacenamiento de gas en el mundo. Puede observarse que Norteamérica y Europa son los mayores usuarios de este tipo de almacenamiento: 359 y 99, respectivamente.

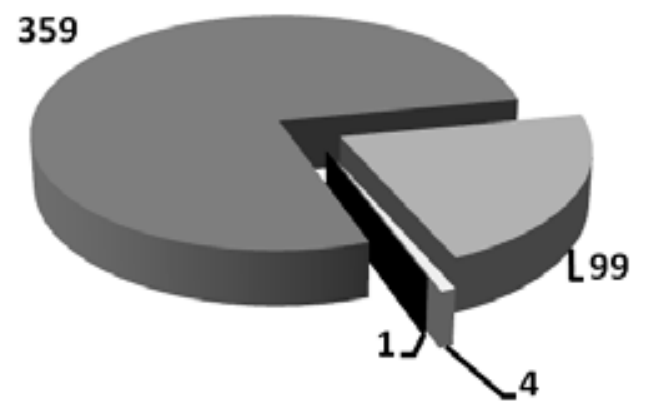

norteamérica

\section{Europa}

Australia

- China

Figura 2. Yacimientos agotados como unidades de almacenamiento DE GAS NATURAL [12]

\section{Aspectos técnicos}

Dentro de los aspectos técnicos la información preliminar es clave para la adecuación de un yacimiento agotado como unidad de almacenamiento de gas natural: geología de la formación, presión inicial del yacimiento, tasas de producción versus presiones [2], temperatura del yacimiento, composición del gas, gravedad específica, número de pozos perforados, localizaciones, profundidades, información de núcleos analizados, mediciones como registros de pozo [13] y pruebas de presión [14] realizadas durante la etapa inicial de producción, estructura del yacimiento, mapas de 
isopacos, si existen mecanismos de empuje de agua, producciones de los pozos, condición mecánica de los mismos, mapa del área del yacimiento y sus vecindades.

Una vez analizada la información preliminar asociada al yacimiento se procede a cálculos y diseños de adecuación de la unidad de almacenamiento de gas teniendo en cuenta que experiencias anteriores en la adecuación de este tipo de yacimientos han mostrado que un incremento de alrededor de un $30 \%$ de la presión inicial de yacimiento es una buena medida de la máxima presión que debe ser usada para su adecuación como unidad de almacenamiento de gas natural [10]. A estas presiones se ha obtenido la máxima capacidad de almacenamiento y la más alta capacidad de flujo para los pozos sin el detrimento de la roca o problemas asociados a la estabilidad de los pozos.

Dentro de las ventajas asociadas a yacimientos agotados está la posibilidad de conocer o estimar fácilmente mediante los históricos de producción la capacidad máxima de almacenamiento [15]; a través de un gráfico de presión de yacimiento sobre factor de compresión (Z) versus producción de gas; indica el gas inicial en el yacimiento y posibles mecanismos de empuje (como agua, etc.) de acuerdo con la forma de la curva. Otras ventajas y desventajas son: muy pocos pozos deben ser perforados, lo que reduce en parte los costos del proyecto; los sistemas de recolección del gas (gas gathering) generalmente ya existen, así como el sistema de conexiones a los gasoductos. La formación geológica ya dispone de un sistema de trampas (preferiblemente anticlinal) para impedir que el gas migre, cuando un yacimiento usado como unidad de almacenamiento de gas natural es abandonado, una significante porción del gas base es no económicamente recuperable, siendo éste uno de los mayores costos involucrados en proyectos de adecuación de yacimientos agotados como almacenes de gas.

En proyectos de yacimientos agotados, cuando el gas base debe ser suministrado a precios presentes, sustanciales reducciones en costo pueden ser obtenidas por el reemplazo de gas natural por un gas base menos costoso [16]. El uso de un gas inerte para 
reemplazar todo o parte del existente gas base del yacimiento resultará en ahorros de valioso gas, el cual podría ser menos cuando éste sea abandonado, además de poseer mayor disponibilidad de gas natural para la venta.

\section{Aspectos económicos}

En general, la distribución de los costos de inversión para la adecuación de yacimientos agotados como unidades de almacenamiento de gas natural es el siguiente [17]: Gas base: $28 \%$, pozos: $24 \%$, compresores: $17 \%$, unidades de deshidratación: $9 \%$, equipos auxiliares: $8 \%$, gasoductos: $7 \%$, edificios: $7 \%$. Según la Internacional Gas Unión, las inversiones de este tipo de almacenamiento podrían suponer entre 0,1 y $0,2 \mathrm{USD} / \mathrm{m}^{3}$, para profundidades entre 1.000 y 2.000 metros y almacenamiento de 1.000 millones de metros cúbicos. Los gastos operativos son del orden de $0,01 \mathrm{USD} / \mathrm{m}^{3}$. En este sentido el costo de adecuación de un yacimiento agotado como unidad de almacenamiento con una capacidad de almacenamiento de 1.000 millones de $\mathrm{m}^{3}$ es del orden de 150 millones de dólares para un precio actual del gas natural de 5 USD/MMBTU equivalente a $0,18 \mathrm{USD} / \mathrm{m}^{3}$ (en promedio 36.000 BTU equivalen a $1 \mathrm{~m}^{3}$ ó 1.020 BTU equivalen a $1 \mathrm{ft}^{3}$ de gas natural).

\subsection{Acuíferos}

La selección de acuíferos como unidades de almacenamiento de gas natural se basa en la geología similar que existe entre los acuíferos y los yacimientos agotados; sin embargo, se debe tener en cuenta que al iniciarse el proyecto la geología y los parámetros de la formación son poco conocidos y el yacimiento está parcial o completamente saturado de agua. Para evaluar la factibilidad del proyecto, se requiere llevar a cabo estudios exploratorios, para definir la capacidad de la estructura y el comportamiento del agua en el acuífero durante la vida del proyecto. Después de los estudios preliminares, se necesitan por lo menos dos pozos en la estructura para desarrollar un programa de ensayos intensivos con el fin de evaluar las propiedades de la formación y la dinámica del acuífero. 
Dentro de las propiedades de los acuíferos adecuados como unidades de almacenamiento de gas, se encuentra la porosidad, en la práctica ésta fluctúa entre un 12 a $25 \%$ [2]. La permeabilidad en una de las rocas más comunes asociadas a los acuíferos, es la lutita (shale) de baja permeabilidad, alrededor de $10^{-4}$ a $10^{-6} \mathrm{mD}$. Típicamente los acuíferos requieren un gas base entre 50 a $80 \%$ [10], el periodo de inyección está entre 200 a 250 días, mientras el de producción entre 100 a 150 días.

En la actualidad existen alrededor de 91 acuíferos adecuados como unidades de almacenamiento de gas natural en el mundo. La figura 3 muestra la distribución por países de los acuíferos adecuados como unidades de almacenamiento de gas natural en el mundo.

- EE.UU.

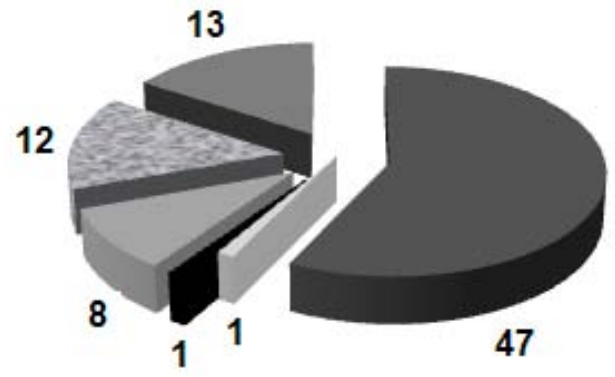

Bélgica

- República Checa

Alemania

Francia

Figura 3. Acuiferos adecuados como unidades de almacenamiento de gas POR PAis [12]

\section{Aspectos técnicos}

Dentro de las diferentes estructuras geológicas de los acuíferos hay una serie de condiciones que el acuífero debe cumplir para la adecuación como unidad de almacenamiento subterráneo de gas natural: Debe haber una estructura bajo la cual el gas es acumulado, capacidad de almacenamiento (porosidad), el espacio para el 
almacenamiento del gas se creará desplazando el agua existente en el espacio poroso, prestando especial atención a la presión de desplazamiento y a las posibles fugas de gas por la disminución de presión, el uso de un acuífero para almacenamiento de gas usualmente requiere más gas base y más monitoreo de las tasas de inyección y de producción que un yacimiento agotado, el contenido volumétrico de gas depende de la configuración estructural, de la porosidad de la roca, de la esperada saturación residual de agua y de la presión usada para el desplazamiento y confinación del gas, un "techo" de roca porosa saturado con agua para impedir que el gas almacenado fluya a través de éste, permitiendo la confinación del gas a altas presiones; la estructura debe proveer una presión de sobrecarga [18] suficiente que permita almacenar gas a presiones muy por encima de la atmosférica, teniendo en cuenta que las presiones de desplazamiento de acuíferos de este tipo están en el orden de 400 a 1500 psi. En este aspecto la profundidad del acuífero es considerada un elemento de importancia en los análisis económicos, ya que el acuífero debe tener una capacidad suficiente para justificar la inversión. Estudios de caso [2] sugieren que el acuífero debe estar a una profundidad mínima de 300 metros, para permitir presiones de fluidos que justifiquen volúmenes de compresión suficientes de acuerdo con cada proyecto, además de tasas de inyección y producción acordes a las necesidades requeridas por el sistema de conexión a los gasoductos, debe haber agua que confine el gradiente de presión en todas las direcciones, algunos inconvenientes que presentan este tipo de almacenamientos son los elevados gastos de exploración, información geológica limitada, la no existencia de gas base inicialmente, la capacidad de la formación es medida únicamente cuando el acuífero sea desarrollado como una unidad de almacenamiento de gas natural, facilidades en superficie así como perforaciones de pozos, operaciones de compresores, zona de deshidratación deben ser diseñadas y realizadas, altas presiones son requeridas para alojar el gas mediante la presión del agua hacia abajo, deshidratación de gas debe ser realizada una vez el gas es producido, más restricciones por las agencias de protección 
del medio ambiente para protección del agua, predicciones de burbujas de agua generadas por la producción de gas una vez esté en funcionamiento. La figura 4 es un esquema donde se muestra los aspectos geológicos fundamentales para la adecuación del acuífero arriba mencionados.

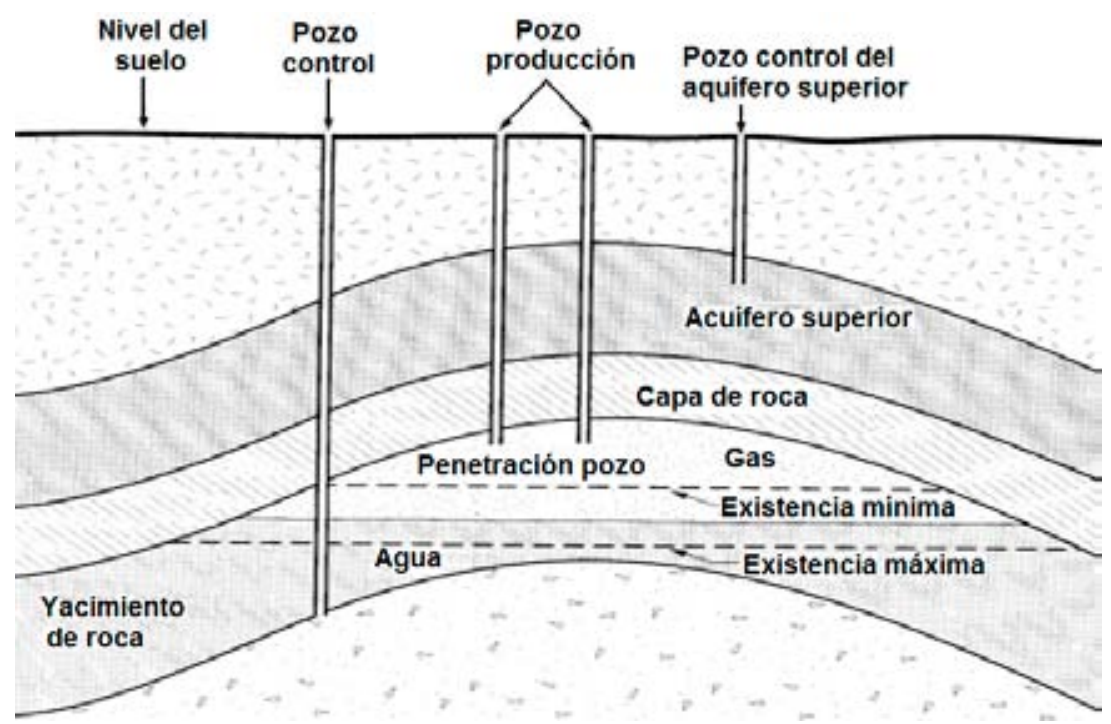

Figura 4. Elementos básicos de un acuífero para almacenamiento del gas [2]

\section{Aspectos económicos de los acuíferos}

En general, la distribución de los costos de inversión es la siguiente [17]:

Gas base: $28 \%$, pozos: $34 \%$, compresores: $14 \%$, unidades de deshidratación: $8 \%$, equipos auxiliares: $6 \%$, gasoductos: $4 \%$, edificios: $6 \%$. Según la Internacional Gas Unión, las inversiones de este tipo de almacenamiento podrían suponer entre 0,2 y $0,3 \mathrm{USD} / \mathrm{m}^{3}$, para profundidades entre $1.000 \mathrm{~m}$ y $2.000 \mathrm{~m}$ y almacenamiento de 1.000 millones de metros cúbicos. Los gastos operativos son del orden de 0,01 USD/m ${ }^{3}$. En este sentido el costo de adecuación de 
un acuífero como unidad de almacenamiento con una capacidad de almacenamiento de 1.000 millones de $\mathrm{m}^{3}$ es del orden de 250 millones de dólares para un precio actual del gas natural de 5 USD/MMBTU equivalente a $0,18 \mathrm{USD} / \mathrm{m}^{3}$ (en promedio $36.000 \mathrm{BTU}$ equivalen a $1 \mathrm{~m}^{3}$ ó $1020 \mathrm{BTU}$ equivalen a $1 \mathrm{ft}^{3}$ de gas natural).

\subsection{Cavernas de sal}

Las cavernas de sal pueden almacenar menos gas que los yacimientos agotados, pero ofrecen más altas tasas de inyección y de producción de gas y por ende un mayor número de ciclos. Este tipo de unidad de almacenamiento es usado principalmente para suplir picos de demanda. Las cavernas de sal para ser adecuadas deben tener la suficiente consistencia y profundidad para soportar las presiones requeridas. El gas base requerido está entre un 20 y $30 \%$, el periodo de inyección está entre 20 y 40 días mientras el periodo de producción está entre 10 a 20 días, un volumen promedio de almacenamiento usado en este tipo de almacenes es alrededor de 500000 metros cúbicos [19]. La figura 5 muestra la distribución por países del número de cavernas de sal adecuadas como unidades. Se observa que Estados Unidos tiene el mayor número de este tipo.

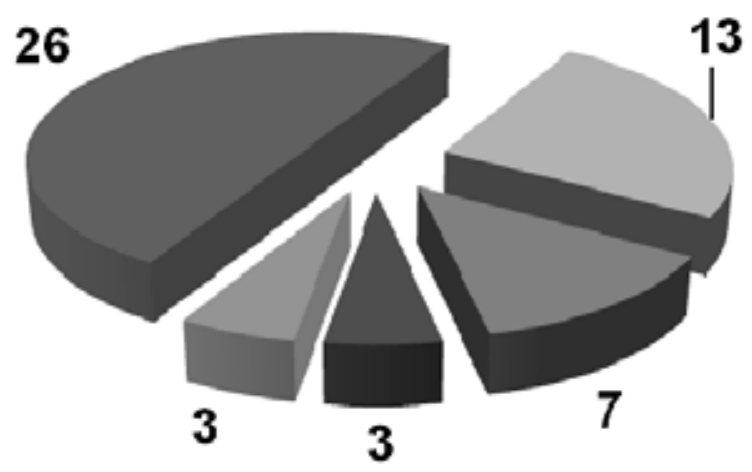

EE.UU.

- Alemania

- Canadá

- Francia

Otros

Figura 5. CAVERnAS dE SAL ADECUADAS COMO UNIDADES DE ALMACENAMIENTO DE GAS NATURAL POR PAIS [12] 


\section{Aspectos técnicos}

El tipo de caverna se hace con base en parámetros de diseño, la capacidad y la presión máxima y mínima del almacenamiento. La primera operación a realizar con el fin de evaluar las propiedades mecánicas de la formación salina es perforar un pozo exploratorio. El pozo exploratorio normalmente se utiliza para los trabajos de lixiviación. Durante la lixiviación el desarrollo de la caverna se controlara mediante modelos matemáticos, basados en pruebas y exploraciones sísmicas.

Una vez la formación de sal es identificada, se taladran pozos y se hace circular agua sobre un intervalo de sal para disolverla como salmuera y posteriormente inyectar el gas que será almacenado [20]. La figura 6 muestra un esquema de una caverna de sal adecuada como almacén de gas. Dentro de las ventajas y

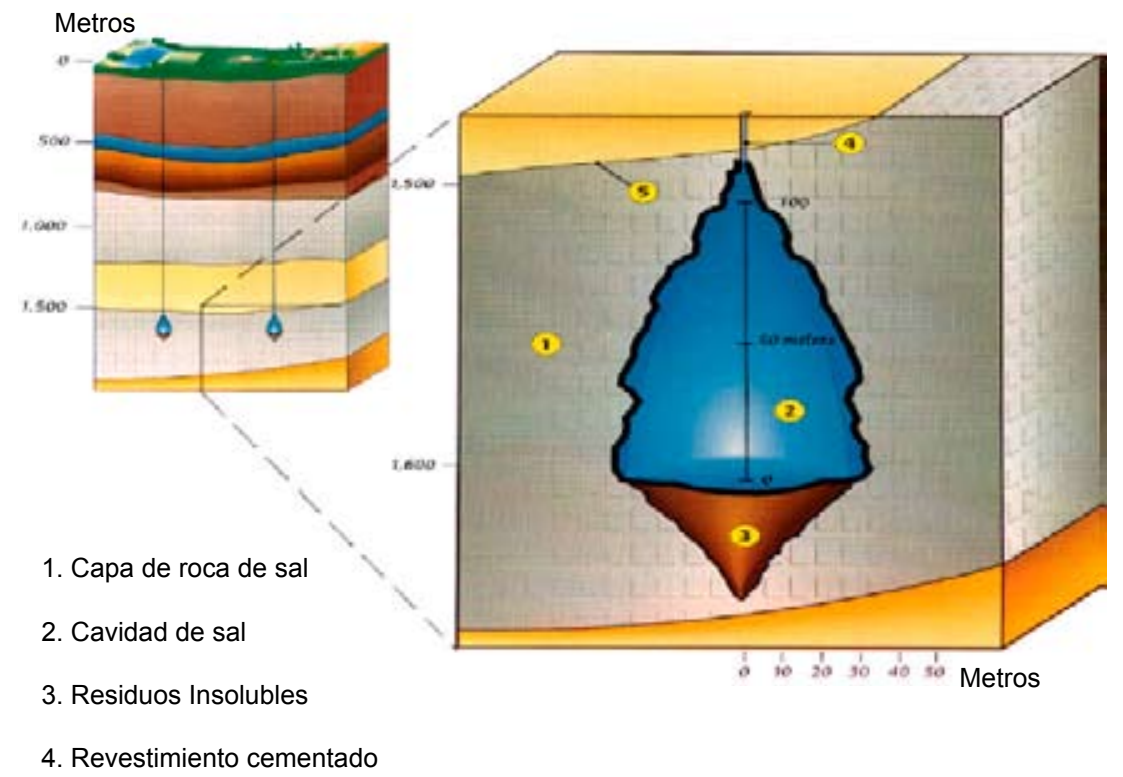

Figura 6. ESQUEMA DE UNA CAVERNA DE SAL ADECUADA COMO UNIDAD DE ALMACENAMIENTO DE GAS 
desventajas de las cavernas de sal están las siguientes: los caudales y las presiones de funcionamiento pueden proyectarse de acuerdo con las necesidades, la productividad de los pozos puede ser 3 ó 4 veces la productividad de los pozos en yacimientos convencionales, la posibilidad de ampliación de la capacidad de almacenamiento mediante lixiviación de cavernas adicionales, el bajo volumen de gas base para elevados caudales de extracción, alto nivel de seguridad, recuperación total del gas base, necesidad de una formación salina idónea, problemas de eliminación de la salmuera en determinadas circunstancias.

\section{Aspectos económicos}

En general, la distribución de los costos de inversión es la siguiente [17]:

Lixiviación: $32 \%$, gas base: $12 \%$, pozos: $15 \%$, compresores: $18 \%$, unidades de deshidratación: $8 \%$, equipos auxiliares: $5 \%$, gasoductos: $4 \%$, edificios: $6 \%$. Las inversiones de este tipo de almacenamiento podrían superar entre 0,4 y $0,5 \mathrm{USD} / \mathrm{m}^{3}$, para profundidades entre 1.000 y 2.000 metros y almacenamientos de 0,5 millones de metros cúbicos. Para las capacidades mencionadas los costos operativos son del orden de $0,01 \mathrm{USD} / \mathrm{m}^{3}$. En este sentido el costo de adecuación de una caverna de sal como unidad de almacenamiento con una capacidad de 0,5 millones de $\mathrm{m}^{3}$ es del orden de 225 millones de dólares para un precio actual del gas natural de 5 USD/MMBTU equivalente a $0,18 \mathrm{USD} / \mathrm{m}^{3}$ (en promedio, 36.000 BTU equivalen a $1 \mathrm{~m}^{3}$ ó 1020 BTU equivalen a $1 \mathrm{ft}^{3}$ de gas natural).

\section{Componentes de una facilidad [21]}

Los componentes en superficie alrededor de los pozos usados para el procesamiento y transporte de gas natural y entendido como facilidad son los siguientes:

Pozos de inyección y producción: Usados para inyectar o producir gas del yacimiento. Debido a las características particula- 
res de cada yacimiento como mecanismos de empuje de la formación no es recomendable, entre otras, inyectar o producir el gas desde un pozo específico. Generalmente los pozos productores son más largos y su diámetro es más grande que pozos normales de producción de un yacimiento no agotado, esto es debido a que más altas tasas de producción son requeridas para almacenaje de gas.

Pozos de observación: Son usados para monitorear la migración de agua y presiones en el yacimiento y deben ser ubicados estratégicamente teniendo en cuenta el tipo de estructura de la formación.

Sistemas de recolección del gas (gathering system): Es el sistema de tuberías que conectan los pozos al punto central de la facilidad.

Compresores: Usualmente localizados en algún punto central cerca a los pozos y pueden ser usados para comprimir el gas para la posterior inyección o producción o ambos.

Sistemas de medición: La tasa de entrega de una facilidad para almacenamiento de gas natural es una variable que depende de la cantidad de gas en el yacimiento en un tiempo determinado, la presión dentro del yacimiento, los sistemas que componen las facilidades, tales como compresores, líneas conectoras a los gasoductos.

Deshidratadores: Un yacimiento siempre contiene agua en alguna proporción, así que cuando gas seco es inyectado y posteriormente producido, éste ha ganado algún grado de agua. El gas deberá ser deshidratado para cumplir con las especificaciones requeridas de venta.

\section{Consideraciones generales}

Cada formación geológica usada como unidad de almacenamiento de gas posee sus propias características físicas y económicas las cuales gobiernan su capacidad para aplicaciones particulares. 
Dentro de las características económicas están la adecuación del sitio, los costos de mantenimiento asociados al buen funcionamiento, las tasas de entrega y el número de ciclos por año que el yacimiento o caverna puede entregar.

Los proyectos de operación de unidades de almacenamiento de gas natural bajo tierra tienen una duración entre 25 y 30 años.Durante la vida de operación, los yacimientos usados como unidades de almacenamiento llegan a estar sujetos a pérdidas que reducen el volumen de gas inventariado, esto debe ser tomado en cuenta en análisis técnicos y económicos. Una incontrolable pérdida de gas asociada a fugas internas representa una menor capacidad de entrega y por ende un flujo de caja menor, además de impactos ambientales negativos. Se hace necesario entonces una auditoría de inventario como variable fundamental durante la operación de este tipo de almacenaje.

La evaluación del desempeño de un yacimiento usado como unidad de almacenamiento de gas involucra el reconocimiento de tres requerimientos básicos llamados atributos [8].

a. Verificación de inventario: Representa el gas que puede almacenar la unidad de almacenamiento y está compuesto de dos volúmenes, gas base (cushion gas) y el gas de trabajo (working gas) el que será producido cuando se requiera.

b. Aseguramiento de los flujos de inyección y de producción de gas natural: Se mide en millones de pies cúbicos estándar y mide la disponibilidad de gas para el mercado.

c. Contenido vs migración: Si las existencias contabilizadas no corresponden a los volúmenes de gas producidos, problemas de migración deberán ser tenidos en cuenta.

El hecho de que el ambiente de almacenamiento está sometido a una presión positiva y siendo el gas natural más liviano que otros fluidos compartiendo el mismo espacio poroso el gas almacenado tiende a migrar. Algunos factores pueden contribuir a la explicación de las diferencias entre el volumen de gas que aparece en libros y los volúmenes totales producidos. Los gradientes de presión, la 
permeabilidad de la roca, la integridad del "techo" de la unidad, geometría, fracturas, fallas, características geológicas, condiciones de operación y limites de los equipos, entre otras.

Para el caso colombiano el suministro de gas natural al sistema nacional de gasoductos proviene de la Guajira con un $90 \%$ y de Cusiana y Cupiagua con un $10 \%$ [22]. Siendo dos ramales los que abastecen el país (la costa Atlántica y el centro del país), ante eventuales fallas o interrupciones inesperadas en los suministros se debe garantizar respaldos ante este tipo de eventos. En esa medida este es un estudio exploratorio del almacenaje de gas natural en el mundo y hace parte del proyecto de tesis de maestría "Pre factibilidad técnica y económica del almacenamiento de gas natural como un sistema de respaldo para el suministro en Colombia”, adscrita a la Maestría en Gestión Energética Industrial del INSTITUTO TECNOLÓGICO METROPOLITANO (ITM).

\section{Biblografía}

[1] BP. (2008) Statistical Review of World Energy. London: BP p.l.c., 2008. p. 22.

[2] KATZ, D. y LEE, R. (1990) Natural Gas Engineering, Production and Storage. Chemical Engineering Series. McGraw-Hill International Editions. 1990. p. 13.

(1990). Natural Gas Engineering, Production and Storage. Chemical Engineering Series. McGraw-Hill International Editions. 1990. p. 493-506.

(1990). Natural Gas Engineering, Production and Storage. Chemical Engineering Series. McGraw-Hill International Editions. 1990. p. 512-541.

[3] TEK, R. (1996) Natural Gas Underground Storage: Inventory and Deliverability. Pen Well. 1996. p. 249-268.

[4] BELCHER, S. (2004) "The Basics of Underground Natural Gas Storage", EIA, 2004.

[5] ROMine, B. (2000). "Real Options in Energy Markets: Analysis and Computation". Thesis PhD. Stanford University. 
[6] THOMPSON, Mathew. (2003) "Real Options \& Optimization of Energy Assets". Thesis, PhD. University of Western Ontario London Ontario Canadá.

[7] DANDEKAR, A. (2006) Petroleum Reservoir Rock and Fluid Properties. Taylor \& Francis. p. 13-26.

[8] LOPERA C., Sergio. (2006). Capítulo 5 Permeabilidad Absoluta, Análisis Petrofísicos Básicos y Especiales, Universidad Nacional de Colombia Sede Medellín, 2006.

[9] FRITZ A., BERGER, HEINZ., BROWN K., FRANTZ J., SAWYER W., HENZELL M., MOHMEYER K., R N., STILES K., XIONG H. (2002) "Storing Natural Gas Underground, Schlumberger”. Oil Field Review, Schlumberger.

[10] FERC (2003) "Current State of and Issues Concerning Underground Natural Gas Storage”, Federal Energy Regulatory Commission Staff Report, September. p. 4.

[11] BROWN K., CHANDLER K., HAWKINS J., MANAI T., ONDERKA V., WALLBRECHT J., ZANGL G. (2008) "Intelligent Well Technology in Underground Gas Storage Oil, Field Review, Schlumberger.

[12] International Human Resources Development Corporation, IHRDC (2008), "The Role of Gas Storage in International Gas Markets". XI Congress of Naturgas. Cartagena Colombia.

[13] TOBY DARLING T. (2005) Well Logging and Formation Evaluation. Elsevier. p. 3-27.

[14] CHAUDHRY A. (2003), Gas Well Testing Handbook. Elsevier. US. p. 237245.

[15] LEE J., WATTENBARGER R., Gas Reservoir Engineering. SPE Text Books Vol. 5. p. 214-229.

[16] MISRA, B.R., FOH, S.E., Inst. Of Gas Technology; Shikari, Y.A., Gas Research Inst; Berry, R.M., Texas Gas Transmission Corporation; Labaune, F., Sofregaz (1988) "The Use of Inert Base Gas in Underground Natural Gas Storage"; SPE 17741, SPE Gas Technology Symposium, Dallas, TX.

[17] SOLÓRZANO HERRERA, I. (2004a). Almacenamiento de Gas Natural en Yacimientos Agotados, Estudio de Viabilidad Y Análisis Económico de un Proyecto desarrollo, Tesis PhD, Escuela Técnica Superior de Ingenieros de Minas. España. p. 42. (2004c). Almacenamiento de Gas Natural en Yacimientos. 
Agotados, Estudio de Viabilidad y Análisis Económico de un Proyecto desarrollo, Tesis PhD, Escuela Técnica Superior de Ingenieros de Minas. España. p. 43.

[18] TIAB DJEBBAR. (2006) Petrophisycs, Theory and Practice of Measuring Reservoir Rock and Fluid Transport Properties. Elsevier. p 53-55.

[19] HOELEN Q., PIJKEREN G., TEUBEN B., STEENBERGEN B., BREUNING P. (2006) Gas Storage in Salt Caverns "AARDGASBUFFER ZUIDWENDING" THE NETHERLANDS. 23 $3^{\text {rd }}$ World Gas Conference, Amsterdam. p. 6.

[20] Gas Processors Suppliers Association (2004). Engineering Data Book. $12^{\text {th }}$ Edición, GPSA. pp. 6-7.

[21] ORIN F. (1995) Underground Gas Storage Facilities, Design and Implementation. Elsevier Science \& Technology Books.

[22] MINISTERIO DE MINAS Y ENERGÍA, Bogotá, Unidad de Planeación Minero Energética UPME (2006). La cadena del gas natural en Colombia. p. 47. 
